\title{
Stochastic thermodynamics analysis of ultrafast AgAu nanoshell dynamics in the nonlinear response regime
}

Guilherme F. Ferbonink, ${ }^{1}$ Thenner S. Rodrigues, ${ }^{2}$ Pedro H.C. Camargo, ${ }^{3}$ Rodrigo Q. de Albuquerque, ${ }^{4}$ and Rene A. Nome ${ }^{1, *}$.

1 Institute of Chemistry, State University of Campinas, Campinas-SP, Brazil

2 Nanotechnology Engineering Program, Alberto Luiz Coimbra Institute for Graduate Studies and Research in Engineering, COPPE, Federal University of Rio de Janeiro, Rio de Janeiro, RJ, Brazil.

3 Department of Chemistry, University of Helsinki, A.I. Vistasen aukio 1, Helsinki, Finland

4 Lehrstuhl für Systemverfahrenstechnik, Technical University of Munich (TUM), Gregor-Mendel-Straße 4, 85354 Freising, Germany.

*nome@unicamp.br

\begin{abstract}
:
The understanding of relaxation dynamics of metallic nanoshells is important for a range of nanotechnological applications. In this work, we present a combined experimental-theoretical study of the relaxation dynamics of $\mathrm{AgAu}$ nanoshells. This was investigated using ultrafast pump-probe experiments resonant with the surface plasmon of the nanoshells, as well as via atomistic molecular dynamics simulations of relaxation and temperature-jump ( $\Delta \mathrm{T}$-jump) processes. Both techniques were then discussed and complemented using a non-equilibrium statistical mechanical model. Data collected at low energies were consistent with our previously reported work and allowed the characterization of intrinsic electron-phonon coupling times (EPCT) and of the overall relaxation dynamics in terms of a two-temperature model. Data at intermediate and higher energies, in turn, showed a nonlinear dependence of EPCT as a function of the pump power, faster relaxation being observed at higher pump energies. In the limit of small $\Delta \mathrm{T}$-jumps, relaxation based on a two-temperature model is recovered, whereas in the limit of large $\Delta \mathrm{T}$-jumps, the relaxation becomes faster with increasing temperature change. The results reported here give insight on the ultrafast dynamics of $\mathrm{AgAu}$ nanoshells and might also be applied to other metallic systems, paving the way to the better understanding of relaxation dynamics of nanoparticles in general.
\end{abstract}




\section{INTRODUCTION:}

One widely used approach to ultrafast time-resolved spectroscopy consists of perturbing the system and recording how it responds as it relaxes back to the equilibrium state. Alternatively, one may look at fluctuations/stochastic dynamics at equilibrium. In the regime of weak perturbation (linear response) these two are connected by the fluctuation dissipation theorem [1,2]. The connection between ultrafast spectroscopy, molecular motion and stochastic dynamics in the linear response regime has been studied in chemical dynamics, solvation, energy transfer, biophysics, among others [3-7]. Given the remarkable developments in fluctuation theorems and far-fromequilibrium thermodynamics [8-11], it is conceptually interesting to see how it can be applied to ultrafast spectroscopy. In the present work, we consider how recent developments in stochastic thermodynamics far-from-equilibrium [12] can be explored to study the ultrafast dynamics of plasmonic nanoparticles in the nonlinear response regime. Recently, this analysis has been explored to study the Markovian MPemba effect manifested in optically trapped colloidal systems [13]. On the other hand, anomalous heating (the inverse effect, which can also described by stochastic thermodynamics far from equilibrium) has been described in nonlinear optical spectroscopy previously as well $[14,15]$. However, to the best of our knowledge, stochastic thermodynamics analysis of ultrafast dynamics of plasmonic nanoparticles in the nonlinear response regime has not been described previously.

Understanding the optical dynamics of plasmonic materials is of current interest to help advance basic knowledge in catalysis and nanoscience, as well as for optimizing their use in applications ranging from nanomedicine to energy [16-19]. Overall, the ultrafast relaxation mechanism of metals is well described by the two-temperature model whereby energy transfer from light to electrons to lattice occurs by absorption, electronic scattering and electron-phonon coupling, respectively, followed by cooling of the atomic lattice and heat transfer to the surrounding environment [20-22]. Additionally for metal nanoparticles, ultrafast impulsive heating also leads to acoustic phonon mode excitation, with subsequent nanoparticle size oscillation and frequencydependent viscous dissipation due to its coupling to the surrounding environment. The models employed to describe heat transfer dynamics and nanomechanical responses observed in ultrafast spectroscopy experiments are based on the fluctuation-dissipation theorem and linear response theory, such that nanoparticle relaxation is linked to 
thermally-induced fluctuations. Thus, the measured ultrafast metal nanoparticle dynamics occurs in the near-equilibrium regime, which is applicable in the limit of weak perturbation by low-intensity pump pulses. Another well characterized regime is that of ultrahigh energy lasers producing joule-level femtosecond pulses interacting with metal nanoparticles. In this pulse energy regime, the metal nanoparticle response is highly nonlinear, and electronic dissipation can be either faster or slower than hydrodynamic cooling, depending on the electron-density, plasma size, and temperature [23-25]. The boundary between the two above mentioned regimes (low and high energy) is regarded as the energy range separating intrinsic nanoparticle dynamics and nonlinear response induced by the pump laser. That is, at low energies, ultrafast spectroscopy experiments as a function of pump power can be used to retrieve the zeropower limit of electron-phonon couplings, thus informing on intrinsic dynamics of the material. At high energies, photo-induced dynamics can differ significantly from that at lower energies, leading for example to hot Brownian motion, structural transitions to degenerate or other nanoparticle shapes, and ablation, among other effects [26-29]. Although the ultrafast dynamics of metal nanoparticles as a function of pump energy has been investigated previously, it is conceptually interesting to connect recent developments in far-from-equilibrium stochastic thermodynamics and the ultrafast dynamics of non-equilibrium nanoparticles in the intermediate energy regime.

Recently, we have reported ultrafast spectroscopy measurements at low pump energies, together with electrodynamics and molecular dynamics (MD) simulations of $\mathrm{AgAu}$ nanoshells with varying composition [30]. The results obtained were correlated with the optical and catalytic properties of the $\mathrm{AgAu}$ nanoshells as a function of composition, showing how ultrafast spectroscopy measurements can inform on catalysis in the absence of light. In the present work, we investigate the resonant ultrafast dynamics of $\mathrm{AgAu}$ nanoshells at intermediate pump pulse energies, in which the relationship between electron-phonon coupling times and pump pulse energy is not linear and non-monotonic. We employ temperature-jump MD simulations to gain atomistic insights into the nanoparticle structural transformations occurring during the pump-probe measurements in this pump energy range. We construct a potential energy landscape informed by the MD results, and then perform far-from-equilibrium stochastic thermodynamics analysis on this energy landscape of temperature-jumpinduced relaxation back to equilibrium. The calculated curves of relaxation time as a 
function of temperature jump are qualitatively consistent with the experimental results. We discuss connections between ultrafast spectroscopy results with the MD and stochastic thermodynamic analysis, and also connect with two-temperature modeling of heat transfer in this system. Overall, the results presented here exemplify the connection between ultrafast spectroscopy measurements of plasmonic nanoparticles beyond the linear-response approximation and stochastic thermodynamics far-from-equilibrium.

\section{MATERIALS AND METHODS:}

\section{Materials and Instrumentation}

Analytical grade silver nitrate $\left(\mathrm{AgNO}_{3}, 99 \%\right.$, Sigma-Aldrich), polyvinylpyrrolidone (PVP, Sigma-Aldrich, M.W. 55,000 g/mol), polyvinylpyrrolidone (PVP, SigmaAldrich, M.W. 10,000 g/mol), ethylene glycol (EG, 99.8\%, Sigma-Aldrich), tetrachloroauric(III) acid trihydrate $\left(\mathrm{HAuCl}_{4.3} \mathrm{H}_{2} \mathrm{O}, \geq 99.9 \%\right.$, Sigma-Aldrich), were used as received.

The scanning electron microscopy (SEM) images were obtained using a JEOL field emission gun microscope JSM 6330F operated at $5 \mathrm{kV}$. The samples were prepared by drop-casting an aqueous suspension containing the nanostructures over a silicon wafer, followed by drying under ambient conditions. UV-VIS spectra were obtained from aqueous suspensions containing the nanostructures with a Shimadzu UV-1700 spectrophotometer. The $\mathrm{Ag}$ and $\mathrm{Au}$ atomic percentages were measured by inductively coupled plasma optical emission spectrometry (ICP-OES) using a Spectro Arcos equipment.

\section{Synthesis of Ag seeds}

$\mathrm{Ag}$ seeds were prepared by the polyol process. In a typical experiment, $5 \mathrm{mg}$ of polyvinylpyrrolidone (PVP) was dissolved in $37.5 \mathrm{~mL}$ of ethylene glycol. Then, $\mathrm{AgNO}_{3}$ (200 mg, $1.2 \mathrm{mmol}$ ) was added and mixed until complete dissolution. The resulting solution was heated to $125{ }^{\circ} \mathrm{C}$ for 2.5 hours, leading to the appearance of a greenishyellow color, allowed to cool down to room temperature, and diluted to $125 \mathrm{~mL}$ of water.

\section{Synthesis of AgAu nanoshells}

The syntheses of $\mathrm{AgAu}$ nanoshells were performed as described previously [31]. The syntheses of $\mathrm{AgAu}$ nanoshells were based on the galvanic replacement reaction between 
performed $\mathrm{Ag}$ nanospheres and $\mathrm{AuCl}_{4}^{-}$(aq) precursor. In a typical run, a mixture containing $5 \mathrm{~mL}$ of PVP (M.W. 55,000 g/mol) aqueous solution $(0.1 \mathrm{wt} \%)$ and $1 \mathrm{~mL}$ of as-prepared suspension containing the $\mathrm{Ag}$ nanospheres was stirred at $100{ }^{\circ} \mathrm{C}$ for $10 \mathrm{~min}$ in a $25 \mathrm{~mL}$ round-bottom flask. Then, $2 \mathrm{~mL}$ of $\mathrm{AuCl}_{4}^{-}$(aq) precursor $(0.8 \mathrm{mM})$ was added dropwise and the reaction allowed to proceed at $100{ }^{\circ} \mathrm{C}$ for another $1 \mathrm{~h}$.

\section{Ultrafast Spectroscopy}

The pump-probe experiments were carried out as described previously [30]. Briefly, 70 fs pulses from a Ti:sapphire laser are split into pump (90\%) and probe (10\%) beams. A $40 \mathrm{~cm}$ focal-length lens was used to focus both pump and probe beams on the sample, and the time delay between beams is controlled by a computer-controlled motorized delay stage. The pulse duration was characterized at the sample position by frequencyresolved optical gating. The sample was placed on a manual xyz-linear translation stage, which was kept fixed in position during the ultrafast experiments. To enhance the signal detection sensitivity, we have used a combination of lock-in amplifier and an optical chopper. The measured signals are fitted by a convolution of the material response function with the instrument response function. The response function, in turn, is a convolution of the Heaviside step function with three exponential decays describing electron-electron scattering, electron-phonon coupling, and phonon-phonon relaxation, an exponentially decaying component accounting for the acoustic oscillation component. These parameters are determined by nonlinear least squares fitting.

\section{Molecular Dynamics Simulations}

MD simulations were carried out using the embedded atom model (EAM) [32] and the LAMMPS program [33]. The NVT ensemble was adopted and the system was initially equilibrated at $1 \mathrm{~K}$. All visualizations were done using the VMD program [34] and computations were run in a MacPro computer (24 processors). The composition $\mathrm{Ag}_{46} \mathrm{Au}_{54}$ was simulated to match the nanoparticle composition employed in the experiments as well as the outer/inner radius ratio of 2 for the simulated nanoshells.

\section{Stochastic thermodynamics analysis}

Far-from-equilibrium stochastic thermodynamics analysis of the nonlinear response regime of $\mathrm{AgAu}$ nanoshells following resonant plasmonic excitation was performed using temperature-jump stochastic simulations on a model energy landscape 
and correlating the calculated cooling times as a function of initial temperature jump. Specifically, we have calculated time-evolving non-Boltzmann probability distributions with the Krammers-Fokker-Planck model for Brownian particle diffusion in the energy landscape informed by the MD simulations (Figure 4(top)) [12]. Given that we can represent our energy landscape with several but finite energy minima and the system evolves under a continuous Fokker-Planck diffusion operator, we describe the configuration space by a transition-rate matrix evolving according to the Markovian Liouville equation for the time-dependent probability. The probability to find the Brownian particle in position $x$ at time $t$ in a potential $\mathrm{V}(\mathrm{x})$ evolves according to Markovian dynamics given by equation:

$$
\partial_{t} p(x, t)=\hat{L} p
$$

Initially, we characterized equilibrium distributions as a function of temperature for the energy landscape employed in this work. Then we calculated non-equilibrium distributions following temperature jump, monitoring the probability distributions as they relaxed back to the equilibrium distribution. In order to quantify non-equilibrium relaxation, we calculate the entropic distance function by:

$$
D_{e}\left[\vec{p} ; T_{b}\right]=\sum\left(\frac{E_{i} \Delta p_{i}}{T_{b}}+p_{i} \ln p_{i}-\pi_{i}^{b} \ln \pi_{i}^{b}\right)
$$

where $\pi_{i}^{b} \sim$ is the equilibrium probability (Boltzmann) distribution, and $\Delta p_{i}=p_{i}-\pi_{i}^{b}$. To characterize the equilibrated system after temperature-jump-induced non-equilibrium perturbation, we defined the cooling time as a lower-bound threshold value for the entropic distance function, which was kept constant as we varied the temperature jump in each simulation. Although this definition of entropic distance threshold is arbitrary, the results and conclusions presented below were insensitive to this threshold. 


\section{RESULTS AND DISCUSSION:}
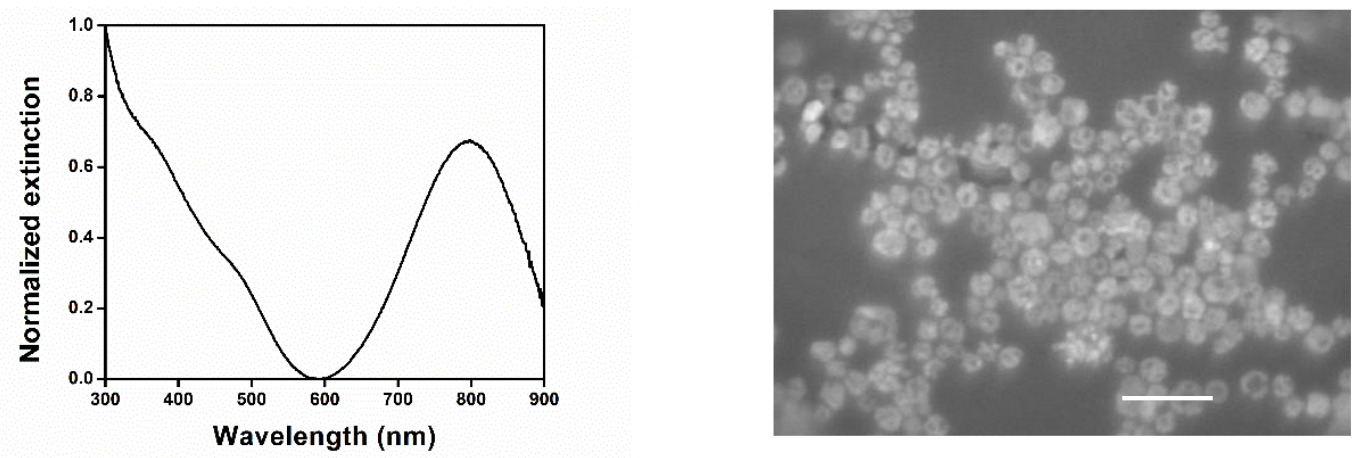

Figure 1. Left: UV-VIS extinction spectrum recorded from aqueous suspensions containing the $\mathrm{AgAu}$ nanoshells obtained from $0.8 \mathrm{mM}$ concentration of a $\mathrm{AuCl}_{4}{ }^{-}(\mathrm{aq})$ solution in the galvanic reaction. Right: SEM image for AgAu nanoshells obtained by the galvanic replacement reaction between Ag NPs (A) and concentrations of $2 \mathrm{~mL}$ of a $\mathrm{AuCl}_{4}^{-}(\mathrm{aq})$ after $1 \mathrm{~h}$ following the addition of $\mathrm{AuCl}_{4}^{-}(\mathrm{aq})$ to a suspension containing $\mathrm{Ag}$ NPs and PVP. The $\mathrm{AuCl}_{4}^{-}(\mathrm{aq})$ concentration corresponded to $0.8 \mathrm{mM}$. Scale bar $=50$ nm.

Figure 1(left) shows the experimental UV-Vis extinction spectrum of the $\mathrm{AgAu}$ nanoshell samples used in this work, which exhibit localized dipolar surface plasmon resonance centered around $800 \mathrm{~nm}$. This SPR wavelength extends well beyond the SPR wavelength of pure $\mathrm{Ag}(410 \mathrm{~nm})$ and $\mathrm{Au}$ nanospheres $(520 \mathrm{~nm})$ or solid $\mathrm{AgAu}$ bimetallic nanoparticles (SPR wavelength intermediate between 410 and $520 \mathrm{~nm}$, depending on composition), due to a complex interplay of structural, compositional and electronic factors that affect their optical properties [30,35]. Figure 1(right) shows electron microscopy images of the $\mathrm{AgAu}$ nanoshells investigated in the present work. The nanoparticles exhibit a hollow core and a diameter of approximately $20-30 \mathrm{~nm}$. The overall size and shape are consistent with the chemical composition of our $\mathrm{AgAu}$ nanoshells employed in our previous work (sample $\mathrm{Au}(0.8)$ ). 

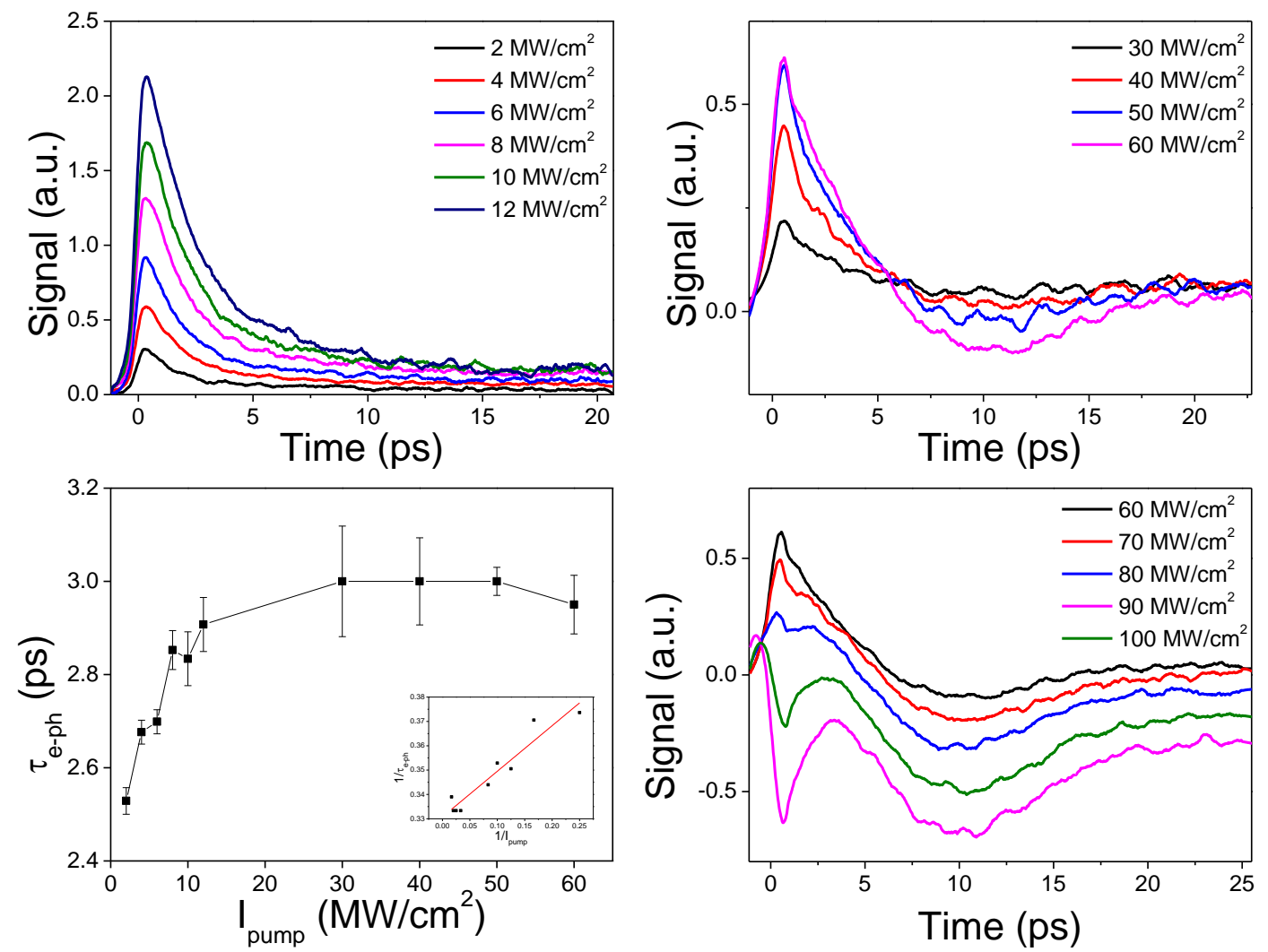

Figure 2. The measured data set is separately plotted in three pump intensity ranges, which denote low energy (top, left, [30]), intermediate energy (top, right) and higher energy (bottom, right). Note that intermediate and higher energy regimes exhibit distinct nonlinear dependence of electron-phonon coupling times on pump energy (bottom, left). The inset in (bottom, left) shows the linearized electron-phonon coupling times vs input pump intensity. In the present work, we focus on the low to intermediate intensity regime with input intensity up to $60 \mathrm{MW} / \mathrm{cm}^{2}$.

Figure 2 shows the pump-probe data measured as a function of pump intensity in the full pump power explored in the present work. Given the large pump power range explored, Figure 2 shows the data set in three distinct ranges, which we denote as low, intermediate, and high pump power. The overall time profile is consistent with previous reports in the literature as well as with our own previous work on the same system. The basic relaxation mechanisms used to describe the temporal profile shown in Figure 2, also discussed previously, involve light absorption by the nanoparticle at the SPR resonance wavelength, leading to coherent electronic motion that decays within a few optical cycles. Electron-electron scattering leads to a hot electron thermal distribution, which transfers thermal energy to the lattice within a few picoseconds by 
electron-phonon coupling. The fast decay results in impulsive lattice heating, which, due to the nanoparticle size, excites acoustic nanoparticle modes with oscillation periods characteristic of the nanoparticle geometry and consistent with continuum mechanics analysis at the nanoscale. Finally, acoustic and phonon relaxation transfer energy to the surrounding medium, thus leading to environment temperature increase. Overall, our microscopy and spectroscopy (steady-state and femtosecond timeresolved) characterization of the $\mathrm{AgAu} 0.8$ nanoshells is consistent with our previous work and with the characteristic ultrafast optical dynamics of plasmonic nanoparticles.

The data shown in Figure 2 (top) was measured in the low pump power range (from 2 to $12 \mathrm{MW} / \mathrm{cm}^{2}$ ), where a linear dependence of electron-phonon coupling times with pump power is obtained [30]. This energy range is commonly employed in order to characterize intrinsic electron-coupling dynamics of the nanoparticle in the absence of light. Pump pulse intensity dependence studies as a function of chemical composition was used to study how electron-phonon coupling time varies as a function of AgAu nanoshell chemical composition. The ultrafast relaxation dynamics of metals and metal nanoparticles has been investigated previously for a range of systems and configurations [20, 36, 37]. A two-temperature model (TTM) can be used to describe the thermal dynamics, where the absorbed pulse energy is used to calculate the initial electron temperature (after light absorption) based on the sample electronic heat capacity. The TTM is also further expanded in different ways: three temperature model, which is still based on heat laws; and integration of TTM with MD in order to obtain a mechanistic description of cooling, especially the observation of acoustic modes at lower frequencies during nanoparticle relaxation [38-41]. Overall, this body of work describes ultrafast dynamics of plasmonic nanoparticles at low pump-pulse pulse intensity, such that the fluctuation-dissipation theorem and linear response regime of non-equilibrium statistical mechanics holds.

On the other hand, the time profiles shown in Figure 2 (top right, and bottom right) exhibit a nonlinear dependence of electron-phonon coupling times with increasing pump power. In Figure 2 (top right), the basic relaxation mechanisms of plasmonic nanoparticles are observed, although with nonlinear dependence of electronphonon coupling times as the pump power increases, see Figure 2 (bottom, left). The results are consistent with previous reports of ultrafast dynamics measurements of plasmonic nanoparticles beyond the linear response regime and before the high energy 
regime [29,42]. The present work focuses on the ultrafast dynamics of $\mathrm{Ag}_{46} \mathrm{Au}_{54}$ nanoshells at low to intermediate pump pulse intensity, for which the electron-phonon coupling times exhibit a non-monotonic intensity dependence, and thus correspond to the nonlinear response regime, as shown in Figure 2 (bottom, left).

On the other hand, above $60 \mathrm{MW} / \mathrm{cm}^{2}$, Figure 2 (bottom, right) shows a highly nonlinear dependence of the time-domain profiles as a function of pump power. This result is qualitatively different from that shown in Figure 2 (top, right), and exemplifies the effects of intermediate versus high pump power on nanoparticle relaxation dynamics following resonant optical excitation. A complex interplay of nonlinear responses, in addition to the nonlinear intensity dependence of electron-phonon coupling times, is observed above $60 \mathrm{MW} / \mathrm{cm}^{2}$, which is thus beyond the scope of the present work [43-50].

Previously, we have reported spectroscopic characterization of $\mathrm{AgAu}$ nanoshells with steady-state UV-vis and ultrafast spectroscopy, together with electrodynamics as well as molecular dynamics simulations [30]. While the spectroscopic studies showed how nanoscale surface segregation, hollow interiors and porosity affect the surface plasmon wavelength and fundamental electron-phonon coupling times as a function of nanoparticle composition, all-atom molecular dynamics simulations of hollow $\mathrm{AgAu}$ core-shell nanoparticles allowed characterization of nanoparticle stability and equilibrium structures, besides providing atomic level views of nanoparticle surface segregation. In order to make a connection between MD simulations and pump-power dependent ultrafast spectroscopy measurements described in the present work, we perform two series of temperature-dependent MD simulations. First, we increase the temperature gradually, in the sense of reversible adiabatic processes in thermodynamics, and characterize nanoparticle structure and energetics as a function of temperature (Figure 3). Second, we perform temperature-jump MD simulations such that the ensuing perturbation is capable of inducing structural transitions between system energy levels, while also monitoring structure and energetics as the system relaxes back to equilibrium (Figure 4). 


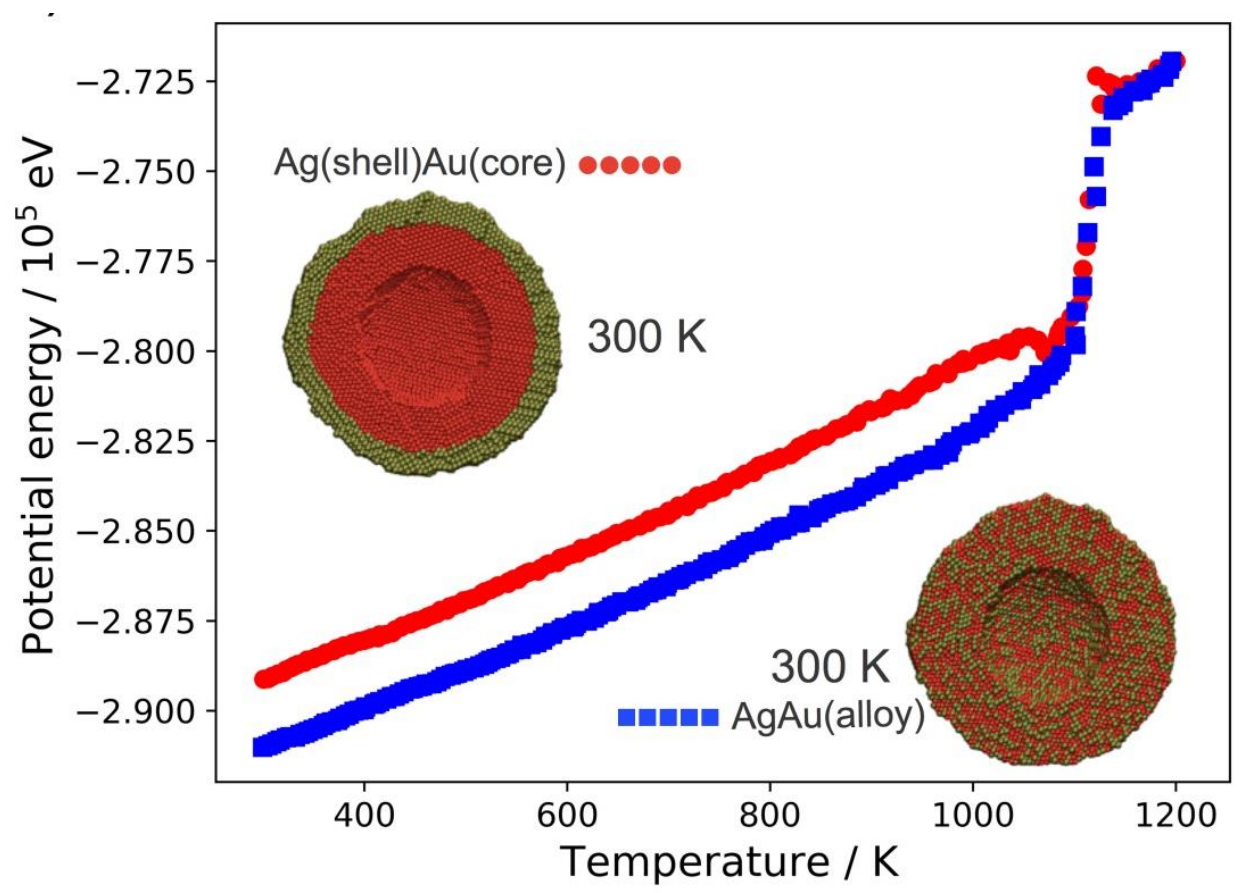

Figure 3. Melting curves for alloyed (blue) and Ag shell / Au core (red) nanoshells with $54 \% \mathrm{Au}$. Inset: Snapshots of the nanoshells with $54 \%$ Au at $300 \mathrm{~K}$.

Figure 3 shows our results from atomistic MD simulations for the $\mathrm{Ag}_{46} \mathrm{Au}_{54}$ nanoshell, which has the same composition of the sample employed in the ultrafast spectroscopy experiments. Figure 3 shows the potential energy as a function of temperature, starting at $300 \mathrm{~K}$, running $20 \mathrm{~ns}-$ long simulations at each temperature, and gradually heating up the system up to $1500 \mathrm{~K}$. We have studied two models for the structural arrangement of hollow nanoparticles: $\mathrm{Ag}_{46} \mathrm{Au}_{54}$ core shell (red points), and $\mathrm{Ag}_{46} \mathrm{Au}_{54}$ alloy (blue points). The inset shows MD snapshots of different structures at $300 \mathrm{~K}$. The comparison of both curves in Fig. 3 reveals that the alloyed structure is thermodynamically preferred.

As can be seen from Figure 3, the two potential energy results are qualitatively similar up to $1000 \mathrm{~K}$, with a linear increase in energy with temperature. In the temperature range from $300 \mathrm{~K}$ to $1000 \mathrm{~K}$, the core-shell nanoparticle is slightly higher in energy when compared with the alloyed structure. Experimentally, the synthesized $\mathrm{Ag}_{46} \mathrm{Au}_{54}$ core shell nanoparticles are stable after their preparation, as described previously [31, 51]. Above $1000 \mathrm{~K}$, the two potential energy curves overlap, reflecting a structural transformation to the molten, non-hollow nanoparticle alloy (not shown), which is the thermodynamically stable arrangement for the $\mathrm{Ag}_{46} \mathrm{Au}_{54}$ system. Analysis of snapshots from the MD simulations with equilibrium structures at different 
temperatures in the temperature range investigated here shows that the nanoparticle maintains an overall core-shell structure up to approximately $1000 \mathrm{~K}$ [30].

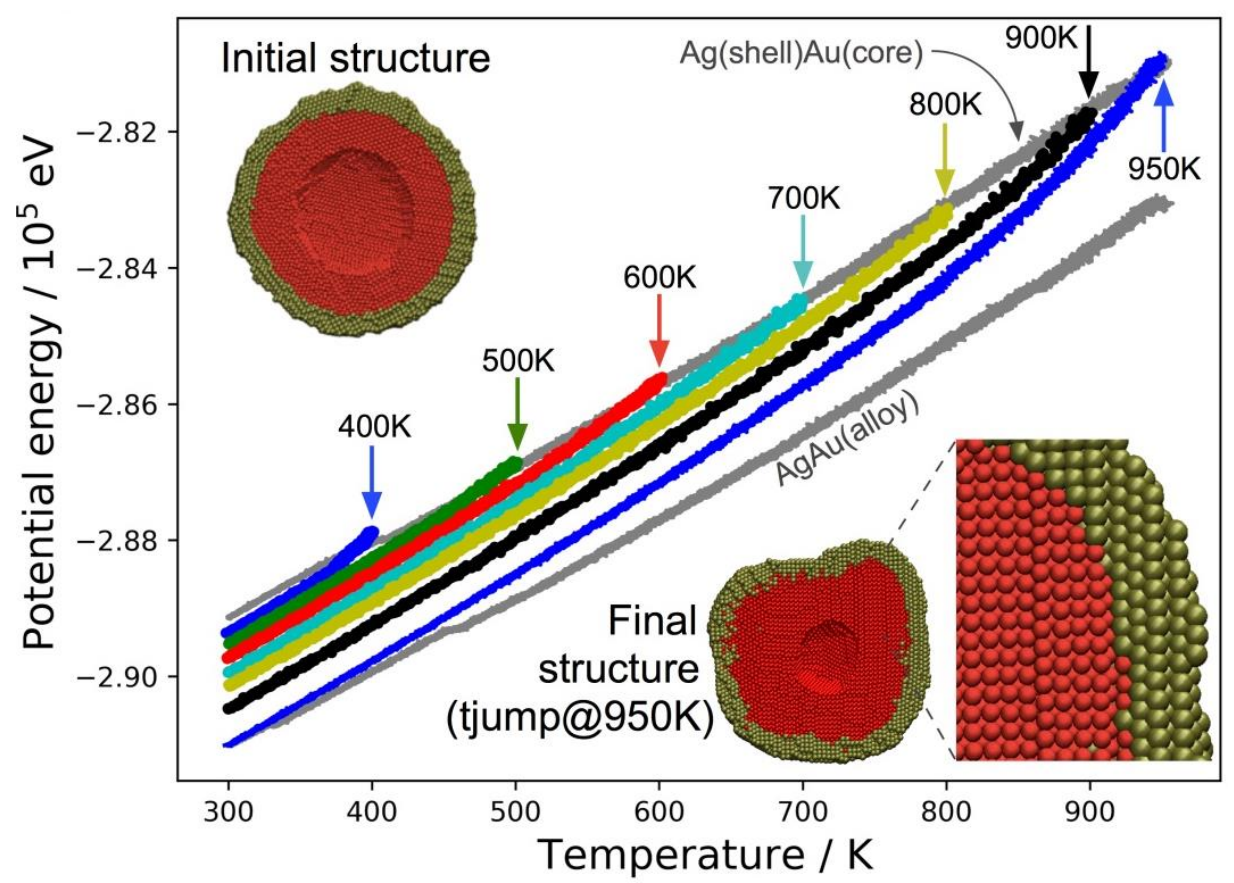

Figure 4. MD simulations of hollow $\mathrm{Ag}$ shell / $\mathrm{Au}$ core nanoparticles with $\mathrm{Ag}_{46} \mathrm{Au}_{54}$ composition: structural and energetic relaxation following temperature-jump ( $\left.\Delta \mathrm{T}_{\mathrm{jump}}\right)$, corresponding to different temperature jumps as indicated by the arrows.

Figure 4 shows potential energy curves as a function of temperature obtained from $\mathrm{MD}$ simulations of $\mathrm{Ag}_{46} \mathrm{Au}_{54}$ nanoshells employing a temperature-jump perturbation protocol $\left(\Delta \mathrm{T}_{\text {jump }}\right)$ at the beginning of each simulation production run. For each simulation, the equilibrated structure starts at $300 \mathrm{~K}$. The perturbation protocol consists of increasing the temperature within a femtosecond by velocity rescaling up to the final temperature, $\mathrm{T}_{\mathrm{f}}$, such that the change in temperature is $\Delta \mathrm{T}_{\mathrm{jump}}=\mathrm{T}_{\mathrm{f}}-\mathrm{T}_{300 \mathrm{~K}}$. At the final temperature $T_{f}$ in the perturbation protocol, the system is relaxed for $1 \mathrm{~ns}$. Subsequently, the system is cooled back to $300 \mathrm{~K}$ using the same procedure described in Figure 3. Temperature-jump molecular dynamics simulations were performed for a set of final temperatures ranging from $T_{\mathrm{f}}=400 \mathrm{~K}$ to $950 \mathrm{~K}$, giving the energy curves shown in Figure 4.

As shown in Figure 4, the final potential energies at $300 \mathrm{~K}$ vary with the magnitude of $\Delta \mathrm{T}_{\text {jump }}$, such that the energies at $300 \mathrm{~K}$ are strongly diminished for larger 
$\Delta \mathrm{T}_{\text {jump }}$, thus indicating that the nanoparticles are more stabilized after the temperaturejump perturbation. Furthermore, by comparing the nanoparticle structures before and after the temperature jump, the size of the cavity also is seen to influence final stability, such that smaller cavity leads to higher stabilization. As shown in the inset of Figure 4, the last structure obtained after T-jump is not alloyed, rather exhibiting a much more stabilized structure with Ag shell-Au core configuration. In comparing Figures 3 and 4 and the MD results obtained for $\mathrm{Ag}_{80} \mathrm{Au}_{20}$ nanoshells reported in [30], analysis of MD snapshots thus shows that atomistic distribution and nanoparticle shape vary with temperature, regardless of the temperature rise protocol employed: slow temperature increase (Figure 3) or temperature-jump (Figure 4). With increasing temperature, atomic mobility increases, thus showing that at higher temperatures more atomic arrangement configurations are thermally accessible, giving rise to a degeneracy that will be explored further below. By further increasing the temperature, core and shell diameter decrease, and upon fusion the nanoparticle becomes partially alloyed (see Supporting Information) and without hollow interior, while phase segregation still occurs. The fused structure at $1200 \mathrm{~K}$ is shown in the SI, with higher Ag surface content. Also, the shell size decreases with increasing temperature, and above $1020 \mathrm{~K}$ the structure coalesces to a solid particle exhibiting alloyed composition ( $\mathrm{Ag}$ is predominant at the surface and $\mathrm{Au}$ at the interior, but both atoms are still somehow mixed, see FIg. SX); however, in the present work we focus on dynamics and relaxation of nanoshells only.

Overall, the MD results presented here provide atomistic insights on the energy landscape for the $\mathrm{Ag}_{46} \mathrm{Au}_{54}$ nanoparticle system, which will be further explored below. Thus, we have calculated a model energy landscape informed by the MD results obtained in the $300 \mathrm{~K}$ to $950 \mathrm{~K}$ range, with multiple energy minima describing composition transformations [52,53] as the system is slowly heated, and an energy barrier leading to a more stable structure, which is accessible when the system is subjected to a temperature jump. These basic features of the energy landscape for the Ag46Au54 system were modeled with the glassy two-state energy landscape shown in Figure 5 (top) [54]. 

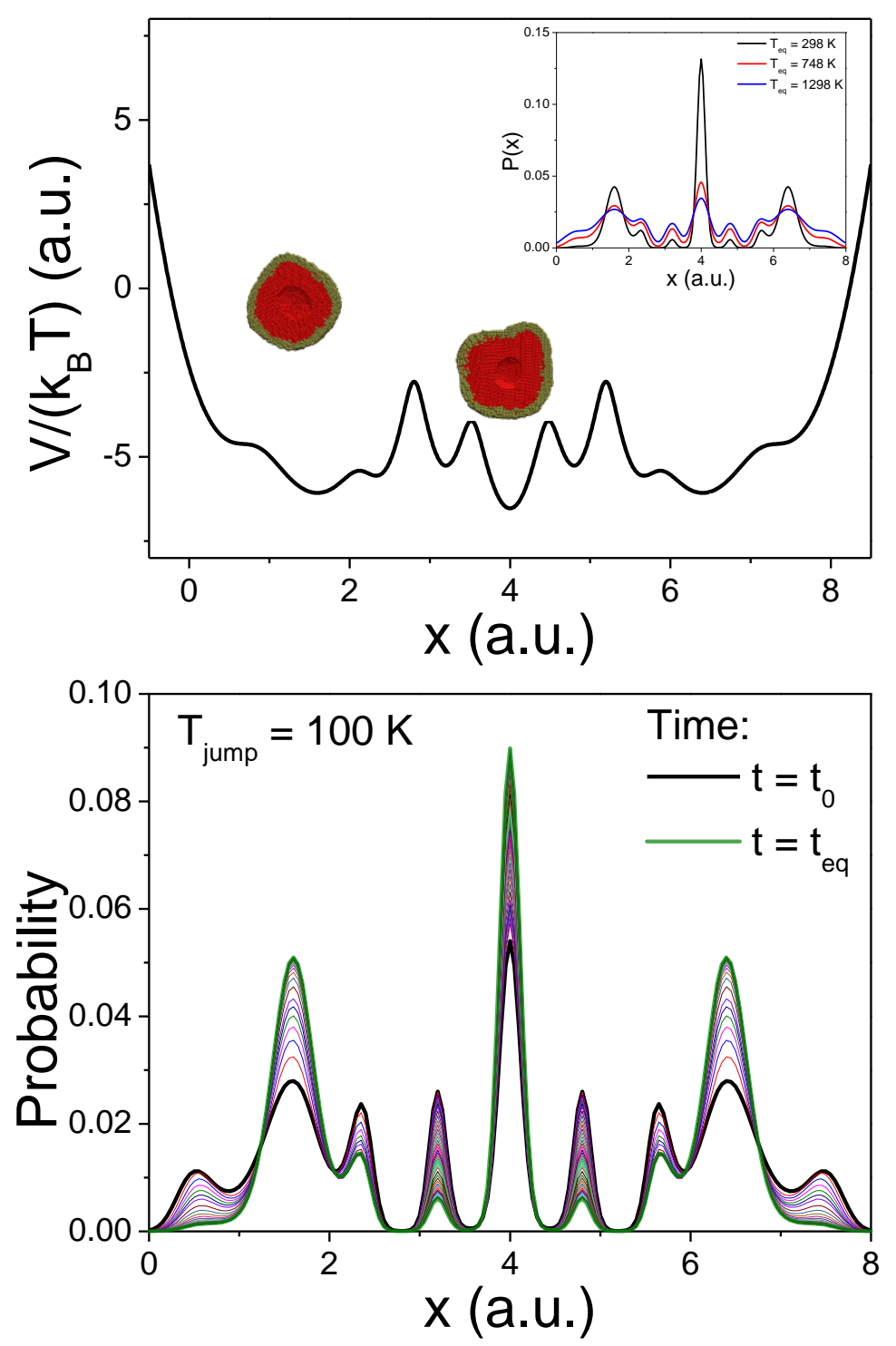

Figure 5. Top: Energy landscape for $\mathrm{Ag}_{46} \mathrm{Au}_{54}$ nanoshells informed by atomistic MD simulations. Structures of the $\mathrm{Ag}_{46} \mathrm{Au}_{54}$ nanoshells, taken from the MD snapshots for different configurations, are shown superimposed in the energy landscape. Inset: Equilibrium probability distributions at three different temperatures. Bottom: Timeevolving probability distributions following temperature-jump $\left(\Delta \mathrm{T}_{\mathrm{jump}}=100 \mathrm{~K}\right)$.

In order to further study the results obtained at intermediate pump energy/temperature-jump reported here, we have performed non-equilibrium statistical mechanical analysis of the relaxation time following temperature-jump perturbation in a model energy landscape. Initially, we have performed equilibrium stochastic dynamics 
simulations on the model energy landscape shown in Figure 5 [53]. Brownian dynamics trajectories at each temperature were used to construct the particle position histogram, which is consistent with the equilibrium Boltzmann probability distributions of finding the particle in different regions of the energy landscape. The Boltzmann distributions were calculated at different equilibrium temperatures. As expected at low temperatures, the Brownian particle spends most of the time in the energy wells whereas at higher temperatures more regions of phase space become thermally accessible (Figure 5 top, inset). Subsequently, we performed non-equilibrium Brownian dynamics simulations following temperature-jump. As shown in Figure 5(bottom), as the system evolves according to equation (1), the initial non-equilibrium probability distribution at $400 \mathrm{~K}$ (black curve in Figure 5, bottom) gradually evolves to the equilibrium Boltzmann distribution at $300 \mathrm{~K}$ (green curve in Figure 5, bottom).
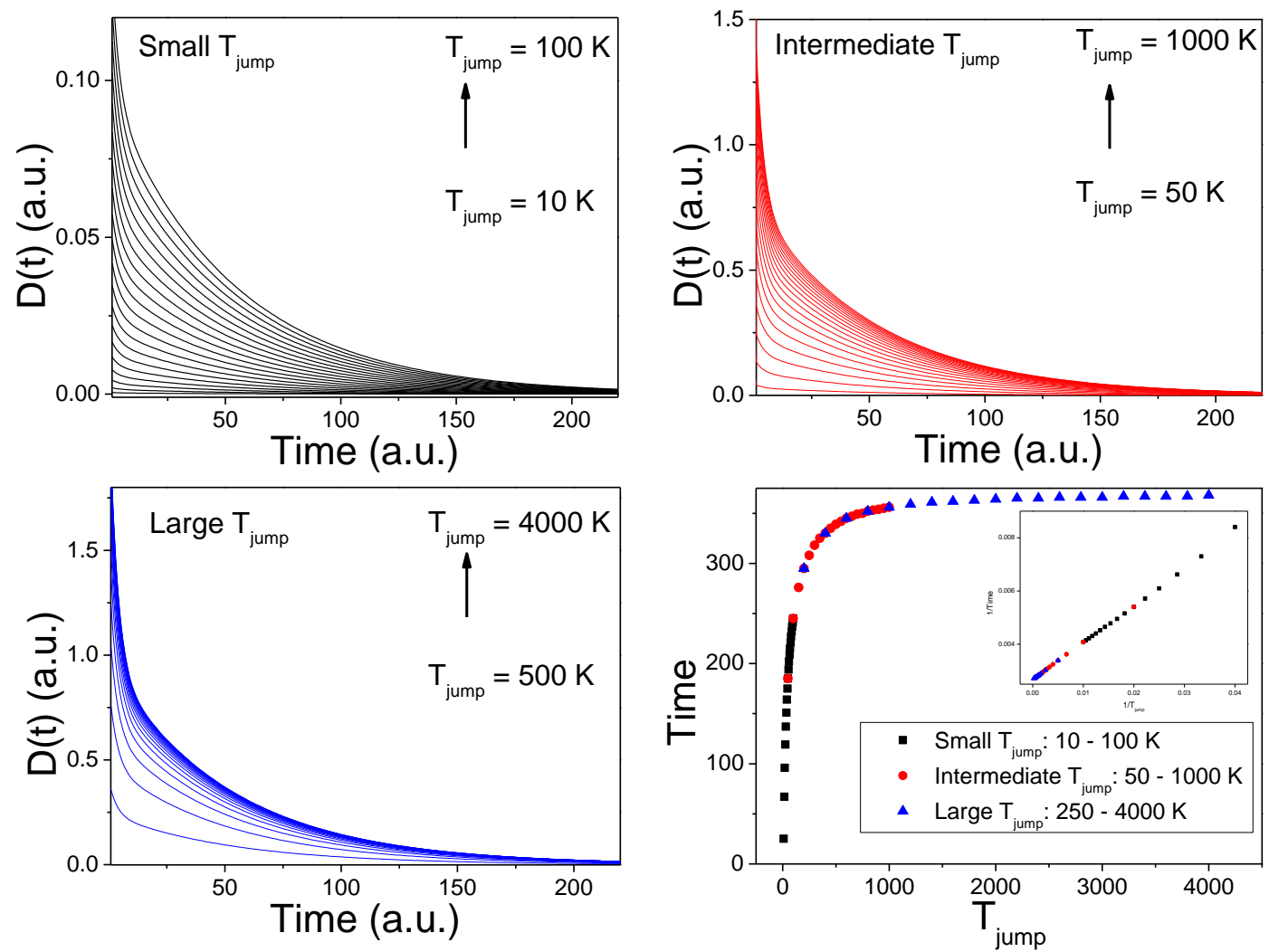

Figure 6 Distance from equilibrium as a function of time for temperature-jump ( $\left.\Delta \mathrm{T}_{\text {jump }}\right)$ values in the $10-100 \mathrm{~K}$ range (top, left figure), 50-1000K (top, right), and 500-4000K (bottom, left). Bottom, right: Cooling time as a function of $\Delta \mathrm{T}_{\text {jump. }}$. The inset shows the linearized plot of cooling times vs $\Delta \mathrm{T}_{\text {jump. }}$. 
Using the same energy landscape shown in Figure 5(top), we have performed simulations as a function of T-jump, calculated time-dependent probability distributions following each T-jump, and calculated the time-dependent entropic distance function, equation (2). The simulations were performed for a range of T-jump values in order to probe the non-equilibrium relaxation dynamics, which are to be compared with results from pump-intensity-dependent ultrafast spectroscopy experiments and T-jump all-atom MD simulations. In the results shown in Figure 6, for each T-jump we have calculated the cooling time as a function of T-jump. Specifically, we define a threshold for the entropic distance function, below which the system is considered to be at equilibrium. The actual threshold value is defined by following the time-evolving probabilities after an impulsive excitation of $5 \mathrm{~K}$, such that the power spectrum of thermal fluctuations is indistinguishable from the true equilibrium state. For all other t-jump simulations investigated in the present work, the cooling time is then defined as the time it takes for the probability distributions - initially prepared at $T_{i}-$ to reach that same threshold value.

Figure 6 shows the relaxation time as a function of initial temperature for a range of initially prepared temperatures. The temperatures chosen are consistent with the pump pulse power, the energy absorbed by the nanoparticle, and the electronic heat capacities of silver and gold. For the energy landscape shown in Figure 5 (top), we thus see two distinct behaviors for the relaxation dynamics. As shown in Figure 6(top, left), at low initial temperatures - still higher than that of the reservoir - relaxation dynamics calculated according to equation (1) shows that higher initial temperatures lead to longer cooling times. On the other hand, by further increasing the initial temperature, a second regime is observed in which cooling time reaches a plateau even though the initial temperature still increases (Figures 6, top right an bottom left). Although qualitatively different from the results based on equilibrium thermodynamics, such "anomalous cooling" effects have been observed in various contexts, including various implementations of the MPemba effect, laser heating of colloidal nanoparticles, and ultrafast dynamics of plasmonic nanoparticles at higher pump-energies. However, we note that a far-from-equilibrium non-equilibrium statistical analysis of the pump-probe data has not been described previously in connection with the ultrafast spectroscopy results. 


\section{CONCLUSIONS:}

In this work, we presented a study of resonant ultrafast dynamics of $\mathrm{AgAu}$ nanoshells as a function of pump intensity at higher energies, together with atomistic molecular dynamics simulations and analysis based on recently developed nonequilibrium statistical mechanical theory. The $\mathrm{AgAu}$ nanoshells were characterized by ICP-MS, scanning electron microscopy and UV-vis spectroscopy, and the overall characterization is consistent with previous reports. Ultrafast pump-probe experiments resonant with the surface plasmon resonance wavelength of the $\mathrm{AgAu}$ nanoshells were performed as a function of pump intensity. Data at low (reported previously) allowed characterization of intrinsic electron-phonon coupling times and the overall relaxation dynamics in terms of a two-temperature model. On the other hand, data at intermediate and higher energies, in turn, showed nonlinear dependence of electron-phonon coupling times as a function of pump-power, with shorter electron-phonon coupling times at higher pump energies. To gain further insights into dynamics at intermediate pump energies, we have performed atomistic molecular dynamics simulations for $\mathrm{AgAu}$ nanoshells with the same elemental composition as in the experiments. The resulting energy landscapes informed by MD were employed to determine equilibrium structures as a function of temperature, thus showing the relative stability of phase-segregated and alloyed $\mathrm{AgAu}$ nanoshells. Moreover, temperature-jump molecular dynamics were simulated in order to compare the relaxation to equilibrium with the experimental ultrafast pump-probe time profiles. Finally, a non-equilibrium statistical mechanical theory of relaxation in a complex energy landscape as a function of temperature perturbation was used to gain further insights into the nature of the $\mathrm{AgAu}$ nanoshell energy landscape and its effect on relaxation dynamics. This analysis was performed with an energy landscape informed by our MD simulations to facilitate comparison with simulation and experimental results. In the limit of small temperature jumps, relaxation based on a two-temperature model is recovered, whereas in the limit of large temperature jumps, the relaxation becomes faster with increasing temperature change. Overall, the results obtained with ultrafast spectroscopy, molecular dynamics simulations and non-equilibrium statistical mechanical analysis are helpful for understanding relaxation dynamics of $\mathrm{AgAu}$ nanoshells in the nonlinear response regime. 


\section{ACKNOWLEDGMENTS:}

We thank Prof. Zhiyue Lu for his assistance and discussions related to the stochastic thermodynamic analysis. Financial support from FAPESP (grant 2016/23430) and $\mathrm{CNPq}$ (the National Council for Scientific and Technological Development) INCT Catalysis in Molecular and Nanostructured Systems is gratefully acknowledged.

\section{REFERENCES:}

[1] Kubo, R., "The fluctuation-dissipation theorem", Rep. Prog. Phys. 29, 255-284 (1966).

[2] Zwanzing, R.W., "Time-correlation functions and transport coefficients in statistical physics", Ann. Rev. Phys. Chem., 16, 67-102 (1965).

[3] Mukamel, S., "Principles of Nonlinear Optical Spectroscopy", Oxford University Press: New York, 1995.

[4] Bixon, M., Jortner, J., "Electron transfer - from isolated molecules to biomolecules", Adv. Chem. Phys. 106, 35-202 (1999).

[5] Nitzan, A. "Chemical Dynamics in Condensed Phases", Oxford University Press: Oxford, 2006.

[6] Fleming, G.R., Cho, M.H., "Chromophore-Solvent Dynamics", Ann. Rev. Phys. Chem. 47 (109), (1996).

[7] Torre, R., "Time-resolved Spectroscopy in Complex Liquids - An experimental perspective", Springer, New York, 2008.

[8] Jarzynski, C., "Nonequilibrium equality for free energy differences" Phys. Rev. Lett., 78 2690.

[9] Seifert, U., "Stochastic thermodynamics, fluctuation theorems and molecular machines", Rep. Prog. Phys., 75, 126001 (2012).

[10] Evans, D. J., Cohen E.G.D., Morriss G.P. "Probability of second law violations in shearing steady states", Phys. Rev. Lett. 71, 24011993.

[11] Sekimoto, K. "Stochastic Energetics: Lecture Notes in Physics", Vol. 799 (Berlin: Springer) (2010).

[12] Z. Lu, O. Raz, "Nonequilibrium thermodynamics of the Markovian Mpemba effect and its inverse," Proc. Nat. Acad. Sci. USA 114, 5083-5088 (2017).

[13] Kumar, A. and Bechhoefer, J. "Exponentially faster cooling in a colloidal system", Nature 584, 64-68 (2020).

[14] Liu, J.M., Kurz, H., Bloembergen, N., "Picosecond time-resolved plasma and temperatureinduced changes of reflectivity and transmission in silicon" Applied Physics Letters 41(7), 643646 (1981).

[15] Fujimoto, J.G., Liu, J.M., Ippen, E.P., Bloembergen, N. "Femtosecond laser interaction with metallic tungsten and nonequilibrium electron and lattice temperatures", Phys. Rev. Lett. 53(19), 1837-1840 (1984).

[16] Arrigo, R., Badmus, Baletto, F., et al "The challenges of characterising nanoparticulate catalysts: general discussion”, Faraday Discussions 208, 339-394 (2018).

[17] Chang, L., Besteiro, L.V., Sun, J., Santiago, E.Y., Gray, S.K., Wang, Z., Govorov, A.O., "Electronic Structure of the plasmons in metal nanocrystals: fundamental limitations for the energy efficiency of hot electron generation" ACS Energy Letters 4(10), 2552-2568 (2019).

[18] Langer, J., de Aberasturi, D.J., Aizpurua, J. et al. "Present and future of surface-enhanced Raman scattering", ACS Nano 14(1), 28-117 (2019).

[19] Nam, J.-W., Liz-Marzán, L., Halas, N., "Chemical Nanoplasmonics: emerging interdisciplinary research field at crossroads between nanoscale chemistry and plasmonics", Accounts of Chemical Research, 52(11), 2995-2996 (2019).

[20] Hartland, G.V., "Optical Studies of Dynamics in Noble Metal Nanostructures," Chem. Rev. 111, 3858-3887 (2011). 
[21] Schoenlein, R. W., Lin, W. Z., Fujimoto, J. G., "Femtosecond studies of nonequilibrium electronic processes in metals", Phys. Rev. Lett., 1987, 58, 1680. 7 P. B. Allen, Phys. Rev.

Lett., 1987, 59, 1460.

[22] Waldecker, L., Bertoni, R., Ernstrofer, R., "Electron-Phonon Coupling and Energy Flow in a Simple Metal beyond the Two-Temperature Approximation", Phys Rev X 6, 021003 (2016).

[23] Rajeev, P. P., Ayyub, P., Bagchi, S., Kumar, G. R., "Nanostructures, local fields, and enhanced absorption in intense light-matter interaction," Opt. Lett. 29, 2662-2664 (2004). [24] Ovchinnikov, A. V., Kostenko, O. F., Chefonov, O. V., Rosmej, O. N., Andreev, N. E., Agranat, M. B., Duan, J. L., Liu, J., Fortov, V. E., "Characteristic x-rays generation under the action of femtosecond laser pulses on nano-structured targets," Laser Part. Beams 29, 249-254 (2011).

[25] Hollinger, R., Bargsten, C., Shlyaptsev, V.N., Kaymak, V., Pukhov, A., Capeluto, M.G., Wang, S., Rockwood, A., Wang, Y., Townsend, A., Prieto, A., Stockton, P., Curtis, A., Rocca, J.J., "Efficient picosecond $\mathrm{x}$-ray pulse generation from plasmas in the radiation dominated regime," Optica 4, 1344-1349 (2017).

[26] Radünz, R. Rings, D., Kroy, K. Cichos, F., "Hot Brownian Particles and Photothermal Correlation Spectroscopy”, J. Phys. Chem. A, 113 (9), pp 1674-1677 (2009).

[27] Merabiaa, S., Shenoginb, S., Jolya, L., Keblinskib, P., Barrata, J.-L., "Heat transfer from nanoparticles: A corresponding state analysis", Proc. Nat. Acad. Sci. USA. 106(36) 1511315118 (2009).

[28] Johns, P., Suess, R.J., Charipar, N., Fontana, J., "Ultrafast Welding Dynamics of Plasmonic Nanorod Dimers", J. Phys. Chem. C 123, 15209-15216 (2019).

[29] De Haan, G., Hernandez-Rueda,J., and Planken, P.C.M., "Femtosecond time-resolved pump-probe measurements on percolating gold in the ablation regime", Optics Express, 28(813), 12093-12107 (2020).

[30] Ferbonink, G.F., Rodrigues, T.S., dos Santos, D.P., Camargo, P.H.C., Albuquerque, R.Q., Nome, R.A., "Correlating structural dynamics and catalytic activity of AgAu nanoparticles with ultrafast spectroscopy and all-atom molecular dynamics simulations," Faraday Discuss. 208, 269-286 (2018).

[31] Slater, T. J. A., Macedo, A., Schroeder, S. L. M., Burke, M. G., O’Brien, P., Camargo, P. H. C., Haigh, S. J., "Correlating Catalytic Activity of Ag-Au Nanoparticles with 3D Compositional Variations", Nano Lett. 14, 1921-1926 (2014).

[32] M. S. Daw and M. Baskes, "Embedded-atom method: Derivation and application to impurities, surfaces, and other defects in metals", Phys. Rev. B: Condens. Matter Mater. Phys., 29, 6443-6453 (1984).

[33] S. Plimpton, "Fast Parallel Algorithms for Short-Range Molecular Dynamics", J. Comput. Phys. 117, 1-19 (1995).

[34] Humphrey, W., Dalke, A., Schulten, K., "VMD - Visual Molecular Dynamics”, J. Mol. Graphics 14, 33-38 (1996).

[35] Goodman, A. M., Cao, Y., Urban, C., Neumann, O., Ayala-Orozco, C., Knight, M. W., Joshi, A., Nordlander, P., N. J. Halas, "Au Nanomatryoshkas as Efficient Near-Infrared Photothermal Transducers for Cancer Treatment: Benchmarking against Nanoshells", ACS Nano, 2014, 8, 6372-6381.

[36] Voisin, C., Del Fatti, N., Christofilos, D., Vallée, F., "Ultrafast Electron Dynamics and Optical Nonlinearities in Metal Nanoparticles", J. Phys. Chem. B 105, 2264-2280 (2001). [37] Park, S., Pelton, M., Liu, M., Guyot-Sionnest, P., Scherer, N.F., "Ultrafast Resonant Dynamics of Surface Plasmons in Gold Nanorods", J. Phys. Chem. C 111, 116-123 (2007). [38] Stoll, T., Maioli, P., Crut, A., Rodal-Cedeira, S., Pastoriza-Santos, I., Vallée, F., Del Fatti, N., "Time-Resolved Investigations of the Cooling Dynamics of Metal Nanoparticles: Impact of Environment”, J. Phys. Chem. C 119, 12757-12764 (2015).

[39] Wang, J., Yu, K., Yang, Y., Hartland, G.V., Sader, J.E., Wang, G.P., "Strong vibrational coupling in room temperature plasmonic resonators", Nat. Commun. 10, 1527 (2019).

[40] Yu, S., Zhang, J., Tang, Y., Ouyang, M., "Engineering Acoustic Phonons and Electron-Phonon Coupling by the Nanoscale Interface", Nano Lett. 15, 6282-6288 (2015). 
[41] Comin, A., Korobchevskaya, K., George, C., Diaspro, A., Manna, L., "Plasmon Bleaching Dynamics in Colloidal Gold-Iron Oxide Nanocrystal Heterodimers", Nano Lett. 12, 921-926 (2012).

[42] Zarick, H.F., Boulesbaa, A., Talbert, E.M., Puretzky, A., Geohegan, D., Bardhan, R., "Ultrafast Excited-State Dynamics in Shape- and Composition-Controlled Gold-Silver Bimetallic Nanostructures" J. Phys. Chem. C 121, 4540-4547 (2017).

[43] Baffou, G., Bordacchini, I., Baldi, A., Quidant, R. "Simple experimental procedures to distinguish photothermal from hot-carrier processes in plasmonics", Light Sci Appl 9, 108 (2020).

[44] Dubi Y., Sivan, Y., "Hot" electrons in metallic nanostructures - nonthermal carriers or heating?", Light Sci Appl 8, 89 (2019).

[45] Hartland, G.V., Besteiro, L.V., Johns, P., Govorov, A.O., "What's so Hot about Electrons in Metal Nanoparticles?" ACS Energy Lett. 2, 1641-1653 (2017).

[46] Melhado, M.S., de Souza, T.G.B., Zilio, S.C., Barbano, E.C., Misoguti, L. "Discrimination between two distinct nonlinear effects by polarization-resolved Z-scan measurements" Optics Express 28(3), 3352-3360 (2020).

[47] Oliveira, N.T.C., Reyna, A.S., Falcão, E.H.L., de Araújo, C.B., "Light Scattering, Absorption, and Refraction due to High-Order Optical Nonlinearities in Colloidal Gold Nanorods" J. Phys. Chem. C, 123, 12997-13008 (2019).

[48] Finot, C., Chaussard, F., Boscolo, S., "Simple guidelines to predict self-phase modulation patterns" J. Opt. Soc. Am. B 35(12), 3143-3152 (2018).

[49] Anna Fitriana,1 Alexander A. Iskandar, AND C. M. de Sterke, "Nonlinear absorption in media with composite gold nanoparticles", J. Opt. Soc. Am. B., 36(11), 2946-2956 (2019). [50] Jauffred, L., Samadi, A., Klingberg, H., Bendix, P.M., Oddershede, L.B., "Plasmonic Heating of Nanostructures" Chem. Rev. 119, 8087-8130 (2019).

[51] Phan, H.T., Haes, A.J., "What Does Nanoparticle Stability Mean?” J. Phys. Chem. C 123, 16495-16507 (2019).

[52] Valencia, F.J., Ramírez, M., Varas, A., Rogan, J., "Understanding the Stability of Hollow Nanoparticles with Polycrystalline Shells”, J. Phys. Chem. C 124, 10143-10149 (2020).

[53] Skorikov, A., Albrecht, W., Bladt, E., Xie, X., van der Hoeven, J.E.S., van Blaaderen, A., van Aert, S., Bals, S., "Quantitative 3D Characterization of Elemental Diffusion Dynamics in Individual Ag@ Au Nanoparticles with Different Shapes" ACS Nano 13, 13421-13429 (2019). [54] Nome, R.A., Costa, A.F., Lepkoski, J., Monteiro, G.A., Hayashi, J.G., Cordeiro, C.M.B., "Characterizing Slow Photochemical Reaction Kinetics by Enhanced Sampling of Rare Events with Capillary Optical Fibers and Kramers' Theory”, ACS Omega 2, 2719-2727 (2017). 


\section{SUPPORTING INFORMATION}
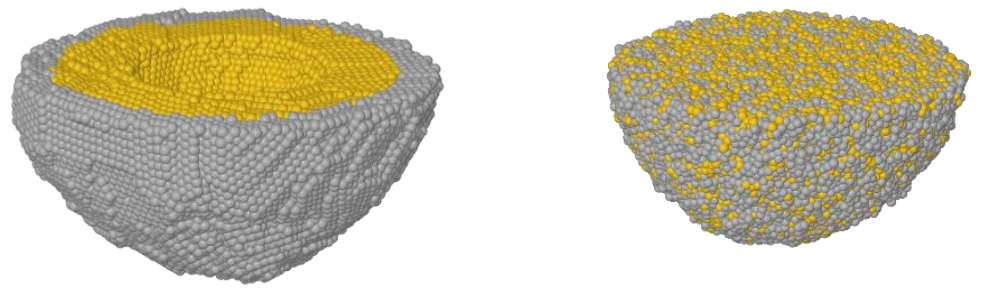

Figure S1. MD Snapshots of equilibrium structures of AgAu nanoparticles at $300 \mathrm{~K}$ (left) and $1200 \mathrm{~K}$ (right). 